Pacific Journal of Mathematics

SUMS OF HILBERT CUBE FACTOR 


\title{
SUMS OF HILBERT CUBE FACTORS
}

\author{
JAMES E. WEST
}

\begin{abstract}
Pursuing the properties of the class of Hilbert cube factors as a sub-class of the compact metric absolute retracts, it is established that the sum theorem for absolute retracts also holds for Hilbert cube factors, that is, a union of two Hilbert cube factors is itself a Hilbert cube factor if the intersection is one. Included is an observation due to T. A. Chapman that the analogous statement is also true for (a certain class of) compact Hilbert cube manifold factors.
\end{abstract}

Over the past several years, the class of Hilbert cube factors, i.e., spaces $X$ with the property that $X \times Y$ is homeomorphic to the Hilbert cube, $Q$, for some space $Y$, has emerged as a significant subclass of the compact metric absolute retracts, appearing in the theory of Hilbert manifolds ([16], [22], [23], [24], [26]), function spaces [16], Hilbert cube manifolds ([9], [10], [11], [12], [13], [22], [23], [25], [26]) and (even) in simple-homotopy theory [12].

It is unknown at present how large a portion of the compact metric absolute retracts is comprised of Hilbert cube factors or even whether there is a compact metric absolute retract which is not a Hilbert cube factor. However, several of the common types of absolute retracts have been shown to be Hilbert cube factors, e.g., contractible polyhedra [22], contractible cell complexes [23], and various hyperspaces ([14], [19], [20], [21], [25]).

Moreover, the class of Hilbert cube factors is known to be closed under several of the standard operations used to construct absolute retracts from others, for example, linite or countably infinite products of Hilbert cube factors are easily seen to be Hilbert cube factors (and countably infinite products of nondegenerate ones are even Hilbert cubes [22]), cones over Hilbert cube factors are Hilbert cube factors [23], and mapping cylinders of maps between Hilbert cube factors are also Hilbert cube factors [23], [26].

The situation with respect to unions of Hilbert cube factors has not been so clear, for although it is not too difficult to show that a union of two absolute retracts which intersect in an absolute retract is an absolute retract [7, p. 90], the only analogous theorems about unions of Hilbert cube factors have presupposed not only that their intersection be a Hilbert cube factor but also that it lie so nicely in one or both of the others that its product with the Hilbert cube, $Q$, is collared in the product with $Q$ of the entire factor or factors (Property $Z$ ), i.e., that $X=F_{1} \cup F_{2}$ is a Hilbert cube factor if $F_{1}, F_{2}$, 
and $F_{1} \cap F_{2}$ are and if $\left(F_{1} \cap F_{2}\right) \times Q$ is collared in both $F_{1} \times Q$ and $F_{2} \times Q$ ([1], implicit in Theorem 10.1) or in one of them ([22], implicit in Theorem 5.1). This paper clarifies the situation by presenting a proof that completely lifts the requirement that the intersection be nicely embedded in either, establishing the Sum Theorem for Hilbert Cube Factors: A union of two Hilbert cube factors is itself a Hilbert cube factor provided that their intersection is one.

Before proceeding, it is worthwhile to clarify the definition and importance of R. D. Anderson's Property $Z$ (a slight generalization of Anderson's original definition [1] is given here). A closed subset, $A$, of an absolute neighborhood retract, $X$, has Property $Z$ in $X$ if the inclusion mapping $U \backslash A \rightarrow U$ is a homotopy equivalence for each open set, $U$, of $X$. (A discussion of this property is contained in [15] which implies, among other things that this definition extends the one originally given in [1], for the case $X=Q$, and that for any absolute neighborhood retract $X$, if $A$ has Property $Z$ in $X$, then $A \times Q$ has Property $Z$ in $X \times Q$.) The reader should regard Property $Z$ intuitively as the topological characterization of "infinite co-dimension" or of "lying in a collared set". (That this is in fact the case inside Hilbert manifolds and Hilbert cube manifolds is demonstrated in [1], [2], [3], [8], and [9].) The latter situation is easily seen to imply Property $Z$ and will be used in the present paper along with the evident fact that if $A$ has Property $Z$ in $X$ and $h: X \rightarrow Y$ is a homeomorphism, then $h(A)$ has Property $Z$ in $Y$. (All homeomorphisms are surjections.)

Property $Z$ is of crucial importance in the homeomorphism theory of infinite-dimensional spaces, as is amply illustrated in the two theorems below which are needed in this paper. The first of these is due to R. D. Anderson [1], and the second, to W. Barit [4] and, independently, to Cz. Bessaga and A. Pelczynski [6]. (See also [5], [21].)

Theorem A (Homogeneity Theorem). Any homeomorphism between two subsets of a Hilbert cube which have Property $Z$ extends to a homeomorphism of the Hilbert cube.

Theorem B (Small Extension Theorem). If $Q$ is a Hilbert cube with chosen metric, then for each $\varepsilon>0$ there is a $\delta>0$ such that any homeomorphism between two subsets of $Q$ with Property $Z$ which moves no point as much as $\delta$ extends to a homeomorphism of $Q$ which moves no point as much as $\varepsilon$.

The next two theorems have been mentioned above, but are important in the proof to follow. 
THEOREM C (Intermediate Sum Theorem [22]). If $X=F_{1} \cup \mathrm{F}_{2}$ is the union of two Hilbert cube factors, $F_{1}$ and $F_{2}$, then $X$ is itself a Hilbert cube factor if $F_{1} \cap F_{2}$ is one and has Property $Z$ in $F_{1}$ or $F_{2}$.

Theorem D (Mapping Cylinder Theorem [23]). If $f: X \rightarrow Y$ is a map between two Hilbert cube factors, then the mapping cylinder, $M_{f}$, of $f$ is also a Hilbert cube factor; moreover, if $c: M_{f} \rightarrow Y$ is the natural collapse of $M_{f}$, then $c \times i d: M_{f} \times Q \rightarrow Y \times Q$ is a uniform limit of homeomorphisms.

(In the above, $M_{f}$ is the quotient space obtained from $X \times$ $[0,1] \cup Y$ by identifying each point, $(x, 0)$, of $X \times\{0\}$ with $f(x)$ in $Y, c$ is the map induced on $M_{f}$ by identifying each point $(x, t)$ of $X \times[0,1]$ with $f(x)$ in $Y$, and " $i d$ " is the identity mapping of the Hilbert cube.)

The main theorem of this paper is concerned with a space $X=$ $F_{1} \cup F_{2}$ which is a union of two Hilbert cube factors, $F_{1}$ and $F_{2}$, which intersect in a third, $F_{3}$. The object is to prove that $X \times Q$ is a Hilbert cube, and the method of proof is to find a sequence $Y_{1}, Y_{2}, \cdots, Y_{n}, \cdots$ of Hilbert cubes, each lying in $X \times Q$, and a sequence of homeomorphisms $h_{\imath}: Y_{\imath} \rightarrow Y_{i+1}, i=1,2, \cdots$, with the property that the successive compositions $h_{\imath} h_{i-1} \cdots h_{1}$ converge to a homeomorphism of $Y_{1}$ onto $X \times Q$. More specifically, let $Q=\prod_{i=1}^{\infty} I_{i}$, where each $I_{i}=[0,1]$, and let $Y_{n} \subset X \times Q$ be the set $F_{1} \times Q \cup F_{2} \times$ $\prod_{i=1}^{n-1} I_{i} \times\{0\}_{n} \times \prod_{i=n+1}^{\infty} I_{2}$. It is a consequence of Theorem $\mathrm{C}$ that each $Y_{n}$ is a Hilbert cube (Lemma 1). For each pair, $m<n$, of positive integers, let $\sigma_{m n}$ be the homeomorphisms of $X \times Q$ which exchanges the $m$ th and $n$th coordinates of $Q$. Then $\sigma_{m n}\left(Y_{m}\right)=Y_{n}$. Moreover, as $n$ becomes large, $Y_{n}$ becomes closer and closer to $X \times Q$ and the sequence $\left\{\sigma_{n n-1} \cdots \sigma_{21}\right\}_{n=2}^{\infty}$ converges uniformly to a map $\sigma$ of $Y_{1}$ onto $X \times Q$. Unfortunately, this map is not a homeomorphism, being essentially the projection "off" the first coordinate of $Q(\sigma(x$, $\left.\left.t_{1}, t_{2}, \cdots\right)=\left(x, t_{2}, t_{3}, \cdots\right)\right)$. This effect is eliminated by interpolating between each pair of $\sigma_{m n}$ 's a "tilting homeomorphism" of the $Y_{n}$ involved and by choosing a subsequence of the $Y_{n}$ 's inductively to guarantee the proper sort of convergence. The construction of these "tilting homeomorphisms" is the primary object of the following lemmas.

In these lemmas, it is convenient for notational purposes to work in the space $X \times I \times J \times K \times Q$, where $I=J=K=[0,1]$ and $Q=\prod_{i=1}^{\infty} I_{i}$ as above. It is also convenient to adopt a specific metric $d$, for $X \times I \times J \times K \times Q$ : Assume a metric $\rho$ for $X$, and let $d$ be given by the formula $d\left(\left(x, a, b, c, t_{1}, t_{2}, \cdots\right),\left(x^{\prime}, a^{\prime}, b^{\prime}, c^{\prime}, t_{1}^{\prime}, t_{2}^{\prime}, \cdots\right)\right)=$ 
$\rho\left(x, x^{\prime}\right)+\left|a-a^{\prime}\right|+\left|b-b^{\prime}\right|+\left|c-c^{\prime}\right|+\sum_{i=1}^{\infty} 2^{-i}\left|t_{\imath}-t_{i}^{\prime}\right|$, where $(x$, $\left.a, b, c, t_{1}, t_{2}, \cdots\right)=\left(x, a, b, c,\left(t_{1}, t_{2}, \cdots\right)\right) \in X \times I \times J \times K \times Q$, etc. (The analogous formula will be used to define metrics, also called $d$, on various sub-products of the factors above, e.g., if $(a, t),\left(a^{\prime}, t^{\prime}\right) \in$ $I \times I_{i}$, then $\left.d\left((a, t),\left(a^{\prime}, t^{\prime}\right)\right)=\left|a-a^{\prime}\right|+2^{-i}\left|t-t^{\prime}\right|.\right)$ Finally, let $Y=F_{1} \times I \times\{0\} \times\{0\} \cup F_{2} \times\{0\} \times\{0\} \times\{0\} \subset X \times I \times J \times K$.

\section{Lemma $1 . \quad Y \times Q$ is a Hilbert cube.}

Proof. $Y \times Q$ is the product with a Hilbert cube of a union of two Hilbert cube factors, $F_{1} \times I \times\{0\} \times\{0\}$ and $F_{2} \times\{0\} \times\{0\} \times\{0\}$, which intersect in a third Hilbert cube factor, $F_{3} \times\{0\} \times\{0\} \times\{0\}$, lying in a collared subset, $F_{1} \times\{0\} \times\{0\} \times\{0\}$, of one of them, so by Theorem $\mathrm{C}$, it is a Hilbert cube.

Let $Z_{1}=Y \cup F_{1} \times\{0\} \times J \times\{0\}$ and let $p_{1}: X \times I \times J \times K \times Q \rightarrow$ $X \times I \times\{0\} \times K \times Q$ be the natural projection.

Lemma 2. For each two positive number, $\varepsilon$ and $\delta$, less than one, there is a homeomorphism, $h$, of $Y \times Q$ onto $Z_{1} \times Q$ which is the identity on $F_{1} \times[\delta, 1] \times\{0\} \times\{0\} \times Q$ such that $h^{-1}$ is within $\varepsilon$ of $p_{1}$.

Proof. $Z_{1}$ may be regarded as the mapping cylinder, $M_{i}$, of the inclusion $i: F_{1} \times\{0\} \times\{0\} \times\{0\} \rightarrow Y$, with $J$ being the parameter of the cylinder. Now, $p_{1} \mid Z_{1} \times Q$ is $c \times i d: M_{\imath} \times Q \rightarrow Y \times Q$, where $c_{2}: M_{i} \rightarrow Y$ is the collapse of $M_{i}$. Therefore, by Theorem $\mathrm{D}, Z_{1}$ is a Hilbert cube factor and $p_{1}: Z_{1} \times Q$ is a uniform limit of homeomorphisms. By Theorem $B$, there is a $\zeta \in(0, \varepsilon / 2)$ such that a homeomorphism between two subsets of $Y \times Q$ which have Property $Z$ extends to a homeomorphism of $Y \times Q$ moving no point as much as $\varepsilon / 2$ provided that it moves no point as much as $\zeta$. Let $f: Z_{1} \times Q \rightarrow Y \times Q$ be a homeomorphism which is within $\zeta$ of $p_{1} \mid Z_{1} \times Q$. Now since $F_{1} \times$ $\{1\} \times\{0\} \times\{0\} \times Q$ is collared in $Z_{1} \times Q$ and in $Y \times Q$, it has Property $Z$ in each of them. Moreover, since $f$ is a homeomorphism, $f\left(F_{1} \times\right.$ $\{1\} \times\{0\} \times\{0\} \times Q)$ has Property $Z$ in $Y \times Q$. Now $f^{-1} \mid f\left(F_{1} \times\{1\} \times\right.$ $\{0\} \times\{0\} \times Q)$ is a homeomorphism between the two sets moving no point as much as $\zeta$, and so extends to a homeomorphism, $g$, of $Y \times Q$ moving no point as much as $\varepsilon / 2$. The composition $g f$ is then a homeomorphism of $Z_{1} \times Q$ onto $Y \times Q$ which is within $\varepsilon$ of $p_{1} \mid Z_{1} \times Q$ and is the identity on $F_{1} \times\{1\} \times\{0\} \times\{0\} \times Q$, so if $k$ is the homeomorphism of $\left(F_{1} \times[0, \delta] \times\{0\} \times\{0\} \cup F_{2} \times\{0\} \times\{0\} \times\{0\}\right) \times Q$ onto $Y \times Q$ which does nothing but multiply the $I$-coordinate by $1 / \delta$, then $k^{-1} g f k$ is a homeomorphism of $Z_{1} \times Q \backslash F_{1} \times(\delta, 1] \times\{0\} \times\{0\} \times Q$ onto $Y \times Q \backslash F_{1} \times(\delta, 1] \times\{0\} \times\{0\} \times Q$ which is within $\varepsilon$ of $p_{1}$ and is the 
identity on $F_{1} \times\{\delta\} \times\{0\} \times\{0\} \times Q$. The desired homeomorphism, $h$, may be taken to be the inverse of the extension of $k^{-1} g f k$ to $Z_{1} \times Q$ which is the identity on $F_{1} \times[\delta, 1] \times\{0\} \times\{0\} \times Q$.

The next lemma is similar to the preceeding one, and its proof, virtually identical, is omitted. For it, let $Z_{2}=Z_{1} \cup F_{1} \times\{0\} \times J \times$ $K \cup F_{2} \times\{0\} \times\{0\} \times K$, and let $p_{2}: X \times I \times J \times K \times Q \rightarrow X \times I \times J \times$ $\{0\} \times Q$ be the natural projection.

LeMma 3. For each two positive numbers, $\varepsilon$ and $\delta$, less than one, there is a homeomorphism, $h$, of $Z_{1} \times Q$ onto $Z_{2} \times Q$ which is the identity on $F_{1} \times[\delta, 1] \times\{0\} \times\{0\} \times Q$ such that $h^{-1}$ is within $\varepsilon$ of $p_{2}$

Next, let $Z_{3}=F_{1} \times I \times\{0\} \times\{0\} \cup F_{1} \times\{0\} \times J \times K$.

LEMMA 4. There is a homeomorphism of $Z_{2} \times Q$ onto $Z_{3} \times Q$ which is the identity on $\left(F_{1} \times I \times\{0\} \times\{0\} \cup F_{1} \times\{0\} \times J \times\{0\}\right) \times Q$.

Proof. Since $F_{1} \times\{0\} \times J \times\{0\} \cup F_{2} \times\{0\} \times\{0\} \times\{0\}$ is homeomorphic to $Y$, it is a Hilbert cube factor by Lemma 1 . Therefore, both $A=\left(F_{1} \times\{0\} \times J \times K \cup F_{2} \times\{0\} \times\{0\} \times K\right) \times Q$ and $B=F_{1} \times$ $\{0\} \times J \times K \times Q$ are Hilbert cubes. Because $F_{1} \times\{0\} \times J \times\{0\} \times Q$ is a subset of a collared set in each of $A$ and $B$, it has Property $Z$ in each. Theorem A may now be applied to guarantee the existence of a homeomorphism, $f$, of $A$ onto $B$ which is the identity on $F_{1} \times\{0\} \times J \times\{0\} \times Q$. Now $Z_{2} \times Q=A \cup F_{1} \times I \times\{0\} \times\{0\} \times Q$ and $Z_{3} \times Q=B \cup F_{1} \times I \times\{0\} \times\{0\} \times Q$, so $f$ may be extended to the desired homeomorphism, $h$, by setting $h$ to be the identity on $F_{1} \times$ $I \times\{0\} \times\{0\} \times Q$.

Let $W=(I \times\{0\} \times\{0\} \cup\{0\} \times J \times K) \times Q$, and let $q: I \times J \times K \times$ $Q \rightarrow\{0\} \times\{0\} \times K \times Q$ be the natural projection.

LEMMA 5. If $1>\varepsilon>\varepsilon_{1}>\varepsilon_{2}>\cdots>\varepsilon_{n}=0$ and $0=\delta_{1}<\delta_{2}<\cdots<$ $\delta_{n}=1$ are two sequences of numbers, then there is a homeomorphism, $h$, of $W$ onto $\{0\} \times J \times K \times Q$ satisfying the following:

(1) $h$ is the identity on $\{0\} \times J \times[\varepsilon, 1] \times Q$,

(2) $q$ h is within $\varepsilon$ of $q$, and

(3) $h\left(\left[\delta_{i}, \delta_{i+1}\right] \times\{0\} \times\{0\} \times Q\right)=\{0\} \times J \times\left[\varepsilon_{\imath+1}, \varepsilon_{\imath}\right] \times Q$, for $i=$ $1,2, \cdots, n-1$.

Proof. Fix $\varepsilon_{0} \in\left(\varepsilon_{1}, \varepsilon\right)$, and let $n$ be a positive integer so large that $2^{-n}<\varepsilon-\varepsilon_{0}$. Let $Q_{n}=\prod_{i=n+1}^{\infty} I_{i}$. Both of $A=(\{0\} \times\{0\} \times\{0\} \cup$ $\left.\{0\} \times J \times\left\{\varepsilon_{0}\right\}\right) \times Q_{n}$ and $B=\left(\{0\} \times J \times\{0\} \cup\{0\} \times J \times\left\{\varepsilon_{0}\right\}\right) \times Q_{n}$ have Property $Z$ in $Q^{\prime}=\{0\} \times J \times\left[0, \varepsilon_{0}\right] \times Q_{n}$.

Let $f:\{0\} \times Q_{n} \rightarrow J \times Q_{n}$ be the coordinate-shifting homeomorphism 
sending each point $\left(0,\left(t_{n+1}, t_{n+2}, \cdots\right)\right)$ to $\left(t_{n+1},\left(t_{n+2}, \cdots\right)\right)$, and let $f_{1}: A \rightarrow B$ be the homeomorphism which is defined from $f$ on $\{0\} \times$ $\{0\} \times\{0\} \times Q_{n}$ and is the identity on $\{0\} \times J \times\left\{\varepsilon_{0}\right\} \times Q_{n}$. By Theorem A, there is an extension, $\bar{f}_{1}$, of $f_{1}$ to a homeomorphism of $Q^{\prime}$.

Define a homeomorphism, $\bar{f}_{2}$, of $I \times\{0\} \times\{0\} \times Q_{n}$ onto $I \times J \times$ $\{0\} \times Q_{n}$ which changes no point's $I$-coordinate and for each $t$ is defined on $\{t\} \times\{0\} \times\{0\} \times Q_{n}$ exactly as is $f$. Now, $\bar{f}_{1}$ and $\bar{f}_{2}$ agree on $\{0\} \times\{0\} \times\{0\} \times Q_{n}$ and together define a homeomorphism $g$, of $(I \times\{0\} \times\{0\} \cup\{0\} \times J \times K) \times Q_{n}$ onto $(I \times J \times\{0\} \cup\{0\} \times J \times K) \times Q_{n}$ which is the indentity on $\{0\} \times J \times\left[\varepsilon_{0}, 1\right] \times Q_{n}$. By taking the product of $g$ with the identity mapping of $\prod_{i=1}^{n} I_{i}$, a homeomorphism, $g_{1}$, is obtained from $W$ to $(I \times J \times\{0\} \cup\{0\} \times J \times K) \times Q$ which

(1) is the identity on $\{0\} \times J \times\left[\varepsilon_{0}, 1\right] \times Q$,

(2) has the property that $q g_{1}$ is within $\varepsilon$ of $q$, and

(3) carries $\{t\} \times\{0\} \times\{0\} \times Q$ to $\{t\} \times J \times\{0\} \times Q$ for each $t \in I$.

The desired homeomorphism, $h$, may be obtained by following $g_{1}$ with a homeomorphism, $g_{2}$, which is the product with the identity on $Q$ and $J$ of a piecewise linear homeomorphism of $I \times\{0\} \cup\{0\} \times$ $K \subset I \times K$ onto $\{0\} \times K$.

It is now possible to establish the "Tilting Lemma".

Let $p_{3}: X \times I \times J \times K \times Q \rightarrow X \times\{0\} \times J \times K \times Q$ be the natural projection.

Lemma 6 (Tilting Lemma). For any two positive numbers, $\varepsilon$ and $\delta$, less than one, there is a homeomorphism, $h$, of $Y \times Q$ onto itself such that $p_{3}$ h is within $\varepsilon$ of $p_{3} \mid Y \times Q$ and which has the property that if $a$ and $b$ are points of $Y \times Q$ which differ in the I-coordinate by as much as $\delta$, then $p_{3} h(a) \neq p_{3} h(b)$.

Proof. By Lemmas 2 and 3 there is a homeomorphism, $f_{1}$, of $Y \times Q$ onto $Z_{2} \times Q$ which is the identity on $F_{1} \times[\delta / 2,1] \times\{0\} \times\{0\} \times Q$ and, except for the contributions of the $J$ - and $K$-coordinates, moves no point as much as $\varepsilon / 5$. By Lemma 4 , there is a homeomorphism, $f_{2}$, of $Z_{2} \times Q$ onto $Z_{3} \times Q\left(=F_{1} \times I \times\{0\} \times\{0\} \times Q \cup F_{1} \times\{0\} \times J \times\right.$ $K \times Q)$ which is the identity on $F_{1} \times I \times\{0\} \times\{0\} \times Q \cup F_{1} \times\{0\} \times$ $J \times\{0\} \times Q^{\cdot}$ Denote $F_{1} \times\{0\} \times J \times K \cup F_{2} \times\{0\} \times\{0\} \times K$ by $Y^{\prime}$ and observe that $Y^{\prime} \times Q=f_{2}^{-1}\left(F_{1} \times\{0\} \times J \times K \times Q\right)$.

Since $f_{2}$ is the identity on $F_{1} \times\{0\} \times J \times\{0\} \times Q$, there is a $\zeta>0$ such that $\zeta<\varepsilon / 5$ and such that if the $K$-coordinate of a point is less than $\zeta$, then $f_{2}^{-1}$ moves that point by less than $\varepsilon / 5$. Moreover, there is a sequence $\zeta>\zeta_{1}>\zeta_{2}>\cdots>\zeta_{n}>\cdots$ of positive numbers such that $p_{1} f_{2}^{-1}\left(F_{1} \times\{0\} \times J \times\left[\zeta_{n}, 1\right] \times Q\right) \cap p_{1} f_{2}^{-1}\left(F_{1} \times\{0\} \times J \times\left[0, \zeta_{n+1}\right] \times\right.$ $Q)=\varnothing$ for each $n=1,2, \cdots$.

Now let $m$ be the first integer for which $(m-1) \delta / 4 \geqq 1$, choose 
$\delta_{m}=1$, and let $\delta_{i}=(i-1) \delta / 4$ for $i=1, \cdots, m-1$. By Lemma 5, there is a homeomorphism, $g$, of $W \times Q=(I \times\{0\} \times\{0\} \cup\{0\} \times J \times$ $K) \times Q$ onto $\{0\} \times J \times K \times Q$ which (1) is the identity on $\{0\} \times J \times$ $[\delta, 1] \times Q$, (2) has a maximal component of motion in the $K \times Q$ coordinates of less than $\delta$ and (3) carries $\left[\delta_{i}, \delta_{i+1}\right] \times\{0\} \times\{0\} \times Q$ homeomorphically to $\{0\} \times J \times\left[\zeta_{i+1}^{\prime}, \zeta_{i}^{\prime}\right] \times Q$ for each $i=1, \cdots, m$, where $\zeta_{i}^{\prime}=\zeta_{i}$ if $i<m$ and $\zeta_{m}^{\prime}=0$. Let $i d$ be the identity map of $X$ and set $f_{3}: i d \times g: Z_{3} \times Q \rightarrow F_{1} \times\{0\} \times J \times K \times Q$.

The composition $f_{2}^{-1} f_{3} f_{2}$ is then a homeomorphism of $Z_{2} \times Q$ onto $Y^{\prime} \times Q$ with a maximal component of motion in the $X \times K \times Q$ coordinates of less than $3 \varepsilon / 5$ and has by construction the property that if $a$ and $b$ are points of $Z_{2} \times Q$ which differ in the $I$-coordinate by as much as $\delta / 2$, then their images under $f_{2}^{-1} f_{3} f_{2}$ differ in some coordinate other than $J$. Since $f_{1}$ moves only points with $I$-coordinate less than $\delta / 2$, this last condition holds for $f_{2}^{-1} f_{3} f_{2} f_{1}: Y \times Q \rightarrow Y^{\prime} \times Q$ with $\delta$ replacing $\delta / 2$.

The desired homeomorphism, $h$, may be constructed from $f_{2}^{-1} f_{3} f_{2} f_{1}$ by regarding $J$ as $I$ and $K$ as a high-indexed coordinate of $Q$. To be precise, let $\bar{f}_{4}: K \times Q \rightarrow\{0\} \times Q \subset K \times Q$ be a homeomorphism within $\varepsilon / 5$ of the natural projection, let

$$
\begin{aligned}
f_{4}= & i d \times \bar{f}_{4}:\left(F_{1} \times\{0\} \times J \cup F_{2} \times\{0\} \times\{0\}\right) \times(K \times Q) \\
& \longrightarrow\left(F_{1} \times\{0\} \times J \cup F_{2} \times\{0\} \times\{0\}\right) \times(\{0\} \times Q),
\end{aligned}
$$

and let $f_{5}: X \times I \times J \times K \times Q \rightarrow X \times I \times J \times K \times Q$ be the homeomorphism which exchanges the $I$ - and $J$-coordinates. Then $h=f_{5} f_{4} f_{2}^{-1} f_{3} f_{2} f_{1}$ is the desired homeomorphism.

With the Tilting Lemma, it is now a relatively simple matter to establish the Sum Theorem.

Sum Theorem for Hilbert Cube Factors. A union of two Hilbert cube factors which intersect in a third is itself a Hilbert cube factor.

Proof. Adopting the notational conventions already made, $X=F_{1} \cup F_{2}, F_{3}=F_{1} \cap F_{2}$, and each $F_{i}$ is a Hilbert cube factor. Also, $Q=\prod_{i=1}^{\infty} I_{i}$ and a metric for $X \times Q$ and various sub-products is explicitly chosen.

Let $\left\{A_{j}\right\}_{j=1}^{\infty}$ be a partition of the positive integers into infinitely many (pairwise disjoint) infinite sets with each $A_{j}=\left\{i_{j 1}, i_{j 2}, \cdots\right\}$ chosen so that $\sum_{k=1}^{\infty} 2^{-i_{j k}} \leqq 2^{1-j}$. Let $Q_{j}=\prod_{k=1}^{\infty} I_{i_{j k}}$. Then $Q=\prod_{j=1}^{\infty} Q_{j}$.

For each $j$, let $Y_{j}^{\prime}=F_{1} \times Q_{j} \cup F_{2} \times\{0\} \times \prod_{k=2}^{\infty} I_{i_{j k}}$, and let $Y_{j}=$ $Y_{j}^{\prime} \times \prod_{m \neq j} Q_{m} \subset X \times Q$. Since each $Y_{j}^{\prime}$ is homeomorphic to the $Y \times Q$ of Lemma 1, each $Y_{j}^{\prime}$ and each $Y_{j}$ is a Hilbert cube. Whenever $m$ and $n$ are positive integers with $m<n$, let $\sigma_{m n}$ be the homeomorphism of $X \times Q$ which exchanges the $i_{m l}$ st and $i_{n l}$ st coordinates of $Q$. Note that $\sigma_{m n}\left(Y_{m}\right)=\left(Y_{n}\right)$. 
By the Tilting Lemma (Lemma 6) there is for each $j$ and each pair, $\varepsilon$ and $\delta$, of positive constants less than one a homeomorphism, $h_{j}(\varepsilon, \delta)$, of $Y_{j}^{\prime}$ which moves no point's $X$-coordinate by as much as $\varepsilon$ and has the property that two points which diffier in the $I_{i_{j 1}}$-coordinate by as much as $\delta$ have images differing in one of the other coordinates of $Y_{j}^{\prime}$. Therefore, there is a similar homeomorphism, $\tau_{j}(\varepsilon, \delta)$ of $Y_{j}$ which is the product of $h_{j}(\varepsilon, \delta)$ with the identity on each of the $Q_{m}$-coordinates for $m \neq j$.

The proof of this theorem consists of the demonstration that a sequence $\left\{j_{m}\right\}_{m=1}^{\infty}$ of positive integers and sequences $\left\{\varepsilon_{m}\right\}_{m=2}^{\infty}$ and $\left\{\delta_{m}\right\}_{m=2}^{\infty}$ of constants in $(0,1)$ may be inductively selected so that if $\varphi_{m}=$ $\tau_{j_{m+1}}\left(\varepsilon_{m+1}, \delta_{m+1}\right) \sigma_{j_{m} j_{m+1}}: Y_{j_{m}} \rightarrow Y_{j_{m+1}}$, then the sequence of compositions $\left\{\chi_{m}=\varphi_{m} \cdots \varphi_{1}\right\}_{m=1}^{\infty}$ converges to a homeomorphism $\chi$ of $Y_{j_{1}}$ onto $X \times Q$. Before the induction conditions are defined, it should observed that just so long as $j_{m}<j_{m+1}$ for each $m$ and the sequence $\left\{\varepsilon_{m}\right\}_{m=2}^{\infty}$ is summable, the maps $\chi_{m}$ are uniformly Cauchy and so converge to a map $\chi$ which is necessarily surjective because each $Y_{j}$ is $2^{1-j}$-dense in $X \times Q$. Thus, the purpose of the induction is to ensure that $\chi$ be one-to-one.

Regarding the set $\left\{\tau_{j}(\varepsilon, \delta) \mid j=1,2, \cdots, \varepsilon \in(0,1), \delta \in(0,1)\right\}$ as chosen, let $\zeta(j, \varepsilon, \delta)$ be one-quarter the minimal distance in coordinates other than $I_{i_{j 1}}$ between images under $\tau_{j}(\varepsilon, \delta)$ of points of $Y_{j}$ which differ in the $I_{i_{j 1}}$-coordinate by at least $\delta$, i.e., if $p_{j}: X \times Q \rightarrow X \times$ $\Pi_{k=2}^{\infty} I_{i_{j k}}$ and $q_{j}: X \times Q \rightarrow I_{i_{j 1}}$ are the projections, then

$$
\begin{aligned}
\zeta(j, \varepsilon, \delta)= & (1 / 4) \min \left\{d\left(p_{j} \tau_{j}(\varepsilon, \delta)(a), p_{j} \tau_{j}(\varepsilon, \delta)(b)\right) \mid a, b \in Y_{j},\right. \\
& \left.2^{-i_{j 1}}\left|q_{j}(a)-q_{j}(b)\right| \geqq \delta\right\} .
\end{aligned}
$$

Now, let $j_{1}, \varepsilon_{2}$, and $\delta_{2}$ be arbitrary, and choose $\left\{j_{m}\right\}_{m=1}^{\infty},\left\{\varepsilon_{m}\right\}_{m=2}^{\infty}$, and $\left\{\delta_{m}\right\}_{m=2}^{\infty}$ inductively so that

(a) $j_{m}<j_{m+1}$,

(b) $2^{-j_{m+1}}<\eta_{m}$, where

$$
\eta_{m}=(1 / 12) \min \left\{d\left(\chi_{m-1}(a), \chi_{m-1}(b)\right) \mid a, b \in Y_{j_{1}}, d(a, b) \geqq 2^{-m}\right\},
$$

(c) $\varepsilon_{m+1}<\varepsilon_{m} / 2$

(d) $\varepsilon_{m+1}<\zeta\left(j_{m}, \varepsilon_{m}, \delta_{m}\right)$

(e) $\varepsilon_{m+1}<\eta_{m}$, and

(f) $\delta_{m+1}<2^{-j_{m+1} \eta_{m}}$.

As mentioned above, (a) and (c) ensure that $\chi=\lim _{m \rightarrow \infty} \chi_{m}$ exists and is a surjection. To verify that $\chi$ is injective, choose two distinct points $a$ and $b$ of $Y_{j_{1}}$. Now let $m$ be so large that $d(a, b) \geqq 2^{-m}$.

Since $A_{j}$ is chosen so that $\sum_{k=1}^{\infty} 2^{-i_{j k}} \leqq 2^{1-j}$, condition (b) implies that the projections of $\chi_{m-1}(a)$ and $\chi_{m-1}(b)$ into $X \times \Pi_{j=1}^{j_{m+1}-1} Q_{j}$ are at least $8 \eta_{m}$ apart. Therefore, at least one of the following is true: 
(1) for some $j<j_{m+1}, j \neq j_{m}, \chi_{m-1}(a)$ differs from $\chi_{m-\tau}(b)$ in the $Q_{j}$-coordinate,

(2) for some $k>1, \chi_{m-1}(a)$ differs from $\chi_{m-1}(b)$ in the $k$ th coordinate of $Q_{j_{m}}$, $4 \eta_{m}$

(3) the $X$-coordinates of $\chi_{m-1}(a)$ and $\chi_{m-1}(b)$ differ by at least

(4) $\chi_{m-1}(a)$ differs from $\chi_{m-1}(b)$ in the first coordinate of $Q_{j_{m}}$ by at least $4 \eta_{m}$.

If (1) or (2) is true, then since $\varphi_{j_{n}}\left(\varepsilon_{n}, \delta_{n}\right)$ does not affect these coordinates for any $n \geqq m, \chi(a) \neq \chi(b)$. On the other hand, if (3) holds, then by (c) and (e) $\chi(a)$ differs from $\chi(b)$ in the $X$-coordinate. Finally, if (4) holds, then $\sigma_{j_{m} j_{m+1}} \chi_{m-1}(a)$ differs from $\sigma_{j_{m} j_{m+1}} \chi_{m-1}(b)$ by at least $\left(4 / 2^{j_{m+1}}\right) \eta_{m}$ in the first coordinate $\left(I_{i_{j m+1}}\right)$ of $Q_{j_{m+1}}$, which is greater than $\delta_{m+1}$ by $(f)$. Thus, in this case, the projections of $\chi_{m}(a)$ and $\chi_{m}(b)$ into $X \times \prod_{k=2}^{\infty} I_{i_{j m+1} k}$ differ by at last $4 \zeta\left(j_{m+1}, \varepsilon_{m+1}, \delta_{m+1}\right)$. This means that either $\chi_{m}(a)$ differs from $\chi_{m}(b)$ in the $k$ th coordinate of $Q_{j_{m+1}}$ for some $k>1$ or that $\chi_{m}(a)$ differs from $\chi_{m}(b)$ in the $X$ coordinate by at least $4 \zeta\left(j_{m+1}, \varepsilon_{m+1}, \delta_{m+1}\right)$. The former case is the same as (2) above, and in the latter case, condition (d) on the size of $\varepsilon_{m+2}$ together with condition (c) ensure that $\chi(a)$ and $\chi(b)$ differ in the $X$-coordinate. Thus, $\chi$ is a homeomorphism and $X$ is a Hilbert cube factor.

An immediate corollary to the above proof is:

COROLlary 1. There are homeomorphisms of $X \times Q$ onto $F_{1} \times$ $I \times Q \cup F_{2} \times\{0\} \times Q$ involving arbitrarily small displacement of the $X$-coordinate.

Proof. The total $X$-displacement of the homeomorphism $\chi^{-1}$ is no more than $2 \varepsilon_{1}$. (Another way to prove this is to use the Sum Theorem together with Theorem $\mathrm{D}$ as in the proof of Lemma 2.)

The second corollary is a generalization of the Sum Theorem to factors of compact Hilbert cube manifolds which was remarked to the author by T. A. Chapman. It is necessary to remark here that at present it is not known whether a factor $X$ of a Hilbert cube manifold must necessarily have the property that $X \times Q$ is a Hilbert cube manifold and thus Corollary 2 is stated only for spaces with the latter property.

Corollary 2 (Chapman). Let $X=F_{1} \cup F_{2}$, where $F_{1} \times Q, F_{2} \times Q$ and $\left(F_{1} \cap F_{2}\right) \times Q$ are compact Hilbert cube manifolds. Then $X \times Q$ is a Hilbert cube manifold.

Proof. Let $C(X)$ be the cone over $X$. Then $C(X)=C\left(F_{1}\right) \cup C\left(F_{2}\right)$, 
and $C\left(F_{1} \cap F_{2}\right)=C\left(F_{1}\right) \cap C\left(F_{2}\right)$. The cone over any compact metric space is the mapping cylinder of the map from the space to the one-point space, so by an extension of Theorem $\mathrm{D}$ [26], $C\left(F_{1}\right), C\left(F_{2}\right)$, and $C\left(F_{1} \cap F_{2}\right)$ are Hilbert cube factors. The Sum Theorem yields that $C(X)$ is a Hilbert cube factor, so $X \times[0,1) \times Q$ is a Hilbert cube manifold and so is $X \times[0,1] \times Q$, which is homeomorphic to $X \times Q$.

Several questions are suggested by this discussion of Hilbert cube factors. The first is whether or not all compact metric absolute retracts are Hilbert cube factors or Hilbert space factors. It is of interest to note in connection with this that there is a topological vector space, $R^{\infty}$, whose product with each finite-dimensional complete metric absolute neighborhood retract is homeomorphic to an open subset of $R^{\infty}$ [18]. (Here, $R^{\infty}$ is the direct limit of the spaces $R^{n}$ with the natural inclusion maps.) A second problem is whether a compact Hilbert cube manifold factor must necessarily have the property that its product with $Q$ is a Hilbert cube manifold. Finally, in several applications, one has two Hilbert cube factors, $X$ and $Y$, and a map $f: A \rightarrow Y$ where $A$ is a subset of $X$ which is a Hilbert cube factor, and one wishes to conclude that the adjunction space $X \cup_{f} Y$ is a Hilbert cube factor. At present, the best that can be said is that the answer is "yes" if $A$ has Property $Z$ in $X$, for then one may use Theorem A to show that $\left(X \cup_{f} Y\right) \times Q$, which is homeomorphic to $(X \times Q) \cup_{f \times i d}(Y \times Q)$, may be regarded as the product with $Q$ of the mapping cylinder of $f$ and conclude by applying Theorem D.

\section{REFERENCES}

1. R. D. Anderson, On topological infinite deficiency, Mich. Math. J., 14 (1967), 365383.

2. R. D. Anderson and T. A. Chapman, Extending homeomorphisms to Hilbert cube manifolds, Pacific J. Math., 38 (1971), 281-293.

3. R. D. Anderson and J. D. McCharen, On extending homeomorphisms to Fréchet manifolds, Proc. Amer. Math. Soc., 25 (1970), 283-289.

4. W. Barit, Small extensions of small homeomorphisms (Abstract), Notices Amer. Math. Soc., 16 (1969), 295.

5. Cz. Bessaga, Topics from infinite-dimensional topology; $\mathscr{K}$-skeletons and Anderson's Z-sets, Aarhus Universitat, Matematisk Institut, Lecture Notes Series \#18.

6. Cz. Bessaga and A. Pełczynski, Estimated extension theorem, homogeneous collections and skeletons, and their applications to topological classifications of linear metric spaces and convex sets, Fund. Math., 69 (1970), 153-190.

7. K. Borsuk, Theory of Retracts, Polish Scientific Publishers (P.W.N.), Monografie Matematyczne, Vol. 44, Warsaw, 1967.

8. T. A. Chapman, Infinite deficiency in Fréchet manifolds, Trans. Amer. Math. Soc., 148 (1970), 137-146.

9. - On the structure of Hilbert cube manifolds, Comp. Math., 24 (1972), 329353. 
10. T. A. Chapman, Surgery and handle-straightening in Hilbert cube manifolds, Pacific J. Math., 45 (1973), 59-79.

11. Compact Hilbert cube manifolds and the invariance of Whitehead torsion, Bull. Amer. Math. Soc., 79 (1973), 52-56.

12. - Topological invariance of Whitehead torsion, Amer. J. Math., (to appear). 13. D. W. Curtis, Simplicical maps which stabilize to near-homeomorphisms, Comp. Math., 25 (1972), 117-122.

14. D. W. Curtis and R. M. Schori, $2^{X}$ and $C(X)$ are homeomorphic to the Hilbert cube, Bull. Amer. Math. Soc., (to appear).

15. J. Eells, Jr. and N. H. Kuiper, Homotopy negligible subsets, Comp. Math., 21 (1969), 155-161.

16. R. Geoghegan, On spaces of homeomorphisms, embeddings and functions, Topology, 11 (1972), 159-177.

17. D. W. Henderson, Applications of infinite-dimensional manifolds to quotient spaces of complete ANR's, Bull. Acad. Polon. Sci. Ser. sci. math. astr., phys. XIX (1971), 747-753.

18. - A simplicial complex whose product with any ANR is a simplicial complex, General Topology and its Applications, 3 (1973), 81-84.

19. R. M. Schori and J. E. West, $2^{I}$ is homeomorphic to the Hilbert cube, Bull. Amer. Math. Soc., 78 (1972), 402-406.

20. - The hyperspace of nonvoid closed subsets of the closed unit interval is homeomorphic to the Hilbert cube, (in preparation).

21. - Hyperspaces of graphs are Hilbert cubes, (preprint).

22. J. E. West, Infinite products which are Hilbert cubes, Trans. Amer. Math. Soc., 150 (1970), 1-25.

23. - Mapping cylinders of Hilbert cube factors, General Topology and its Applications, 1 (1971), 111-125.

24. - Product of complexes and Fréchet spaces which are manifolds, Trans. Amer. Math. Soc., 166 (1972), 317-337.

25. - The subcontinua of a dendron form a Hilbert cube factor, Proc. Amer. Math. Soc., 36 (1972), 603-608.

26. - Mapping cylinders of Hilbert cube factors II-The relative case, General Topology and its Applications, (to appear).

Received October 27, 1972. Partially supported by NSF Grants GP 28244 and GP33960X.

\section{CORNELL UNIVERSity}





\section{PACIFIC JOURNAL OF MATHEMATICS}

\section{EDITORS}

RICHARD ARENS (Managing Editor)

University of California

Los Angeles, California 90024

R. A. Beaumont

University of Washington

Seattle, Washington 98105
J. DugundJI

Department of Mathematics University of Southern California Los Angeles, California 90007

D. Gilbarg AND J. Milgram Stanford University

Stanford, California 94305

\section{ASSOCIATE EDITORS}
E. F. BECKENBACH
B. H. NeUmanN
F. WOLF
K. YOSHIDA

\section{SUPPORTING INSTITUTIONS}

UNIVERSITY OF BRITISH COLUMBIA CALIFORNIA INSTITUTE OF TECHNOLOGY

UNIVERSITY OF CALIFORNIA

MONTANA STATE UNIVERSITY

UNIVERSITY OF NEVADA

NEW MEXICO STATE UNIVERSITY

OREGON STATE UNIVERSITY

UNIVERSITY OF OREGON

OSAKA UNIVERSITY
UNIVERSITY OF SOUTHERN CALIFOF

STANFORD UNIVERSITY

UNIVERSITY OF TOKYO

UNIVERSITY OF UTAH

WASHINGTON STATE UNIVERSITY UNIVERSITY OF WASHINGTON

AMERICAN MATHEMATICAL SOCIET NAVAL WEAPONS CENTER 


\section{Pacific Journal of Mathematics}

\section{Vol. 54, No. 1 \\ May, 1974}

Ralph K Amayo, Engel Lie rings with chain conditions ..................

Bernd Anger and Jörn Lembcke, Hahn-Banach type theorems for hypolinear

functionals on preordered topological vector spaces ..................

Gregory Frank Bachelis and Samuel Ebenstein, On $\Lambda(p)$ sets ................

Harvey Isaac Blau, Indecomposable modules for direct products of finite

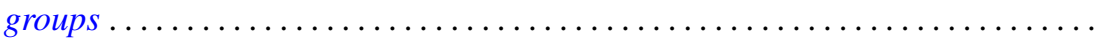

Larry Eugene Bobisud and James Calvert, Singular perturbation of a

time-dependent Cauchy problem in a Hilbert space ................

Walter D. Burgess and Robert Raphael, Abian's order relation and orthogonal

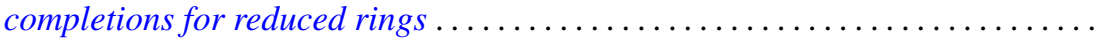

James Diederich, Representation of superharmonic functions mean continuous at

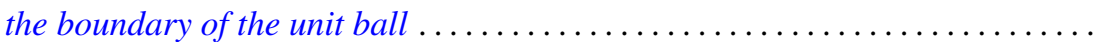

Aad Dijksma and Hendrik S. V. de Snoo, Self-adjoint extensions of symmetric

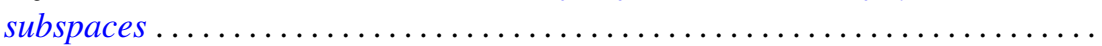

Gustave Adam Efroymson, A Nullstellensatz for Nash rings . . . . . . . . . . . . .

John D. Elwin and Donald R. Short, Branched immersions onto compact orientable surfaces . . . . . . . . . . . . . . . . . . . . . . . . .

John Douglas Faires, Comparison of the states of closed linear

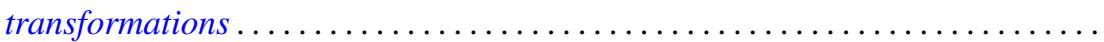

Joe Wayne Fisher and Robert L. Snider, On the von Neumann regularity of rings with regular prime factor rings .

Franklin Takashi Iha, A unified approach to boundary value problems on compact intervals

Palaniappan L. Kannappan and Che Tat $\mathrm{Ng}$, On functional equations connected with directed divergence, inaccuracy and generalized directed divergence

Samir A. Khabbaz and Elias Hanna Toubassi, The module structure of Ext $(F, T)$ over the endomorphism ring of $T$...

Garo K. Kiremidjian, On deformations of complex compact manifolds with boundary.

Dimitri Koutroufiotis, Mappings by parallel normals preserving principal

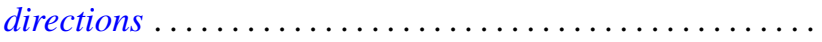

W. K. Nicholson, Semiperfect rings with abelian adjoint group

Norman R. Reilly, Extension of congruences and homomorphisms to translational hulls

Sadahiro Saeki, Symmetric maximal ideals in $M(G)$

Brian Kirkwood Schmidt, On the homotopy invariance of certain functors ...

H. J. Shyr and T. M. Viswanathan, On the radicals of lattice-ordered rings ...

Indranand Sinha, Certain representations of infinite group algebras ...

David Smallen, The group of self-equivalences of certain complexes ...

Kalathoor Varadarajan, On a certain problem of realization in homotopy

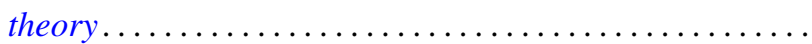

